Article

\title{
Turbulence Intensity and the Friction Factor for Smooth- and Rough-Wall Pipe Flow
}

\author{
Nils T. Basse
}

Toftehøj 23, Høruphav, 6470 Sydals, Denmark; nils.basse@npb.dk

Academic Editor: William Layton

Received: 24 April 2017; Accepted: 8 June 2017; Published: 10 June 2017

\begin{abstract}
Turbulence intensity profiles are compared for smooth- and rough-wall pipe flow measurements made in the Princeton Superpipe. The profile development in the transition from hydraulically smooth to fully rough flow displays a propagating sequence from the pipe wall towards the pipe axis. The scaling of turbulence intensity with Reynolds number shows that the smoothand rough-wall level deviates with increasing Reynolds number. We quantify the correspondence between turbulence intensity and the friction factor.
\end{abstract}

Keywords: turbulence intensity; Princeton Superpipe measurements; flow in smooth- and rough-wall pipes; friction factor

\section{Introduction}

Measurements of streamwise turbulence [1] in smooth and rough pipes have been carried out in the Princeton Superpipe [2-4] (Note that the author of this paper did not participate in making the Princeton Superpipe measurements.). We have treated the smooth pipe measurements as a part of [5]. In this paper, we add the rough pipe measurements to our previous analysis. The smooth (rough) pipe had a radius $R$ of 64.68 (64.92) $\mathrm{mm}$ and a root mean square (RMS) roughness of $0.15(5) \mu \mathrm{m}$, respectively. The corresponding sand-grain roughness is 0.45 (8) $\mu \mathrm{m}[6]$.

The smooth pipe is hydraulically smooth for all Reynolds numbers Re covered. The rough pipe evolves from hydraulically smooth through transitionally rough to fully rough with increasing $R e$. Throughout this paper, Re means the bulk Re defined using the pipe diameter $D$.

We define the turbulence intensity (TI) I as:

$$
I(r)=\frac{v_{\mathrm{RMS}}(r)}{v(r)},
$$

where $v$ is the mean flow velocity, $v_{\text {RMS }}$ is the RMS of the turbulent velocity fluctuations and $r$ is the radius ( $r=0$ is the pipe axis, $r=R$ is the pipe wall).

An overview of past research on turbulent flows over rough walls can be found in the pioneering work by Nikuradse [7] and a more recent review by Jiménez [8].

The development of predictive drag models has previously been carried out using both measurements [9] and direct numerical simulations (DNS) [10]. This work covered the transitionally and fully rough regimes and a variety of rough surface geometries.

The aim of this paper is to provide the fluid mechanics community with a scaling of the TI with $R e$, both for smooth- and rough-wall pipe flow. An application example is computational fluid dynamics (CFD) simulations, where the TI at an opening can be specified. A scaling expression of TI with Re is provided as Equation (6.62) in [11]. However, this formula does not appear to be documented, i.e., no reference is provided. 
Our paper is structured as follows: in Section 2, we study how the TI profiles change over the transition from smooth to rough pipe flow. Thereafter, we present the resulting scaling of the TI with $R e$ in Section 3. Quantification of the correspondence between the friction factor and the TI is contained in Section 4, and we discuss our findings in Section 5. Finally, we conclude in Section 6.

\section{Turbulence Intensity Profiles}

We have constructed the TI profiles for the measurements available (see Figure 1). Nine profiles are available for the smooth pipe and four for the rough pipe. In terms of $R e$, the rough pipe measurements are a subset of the smooth pipe measurements. Corresponding friction Reynolds numbers can be found in Table 1 in [3].

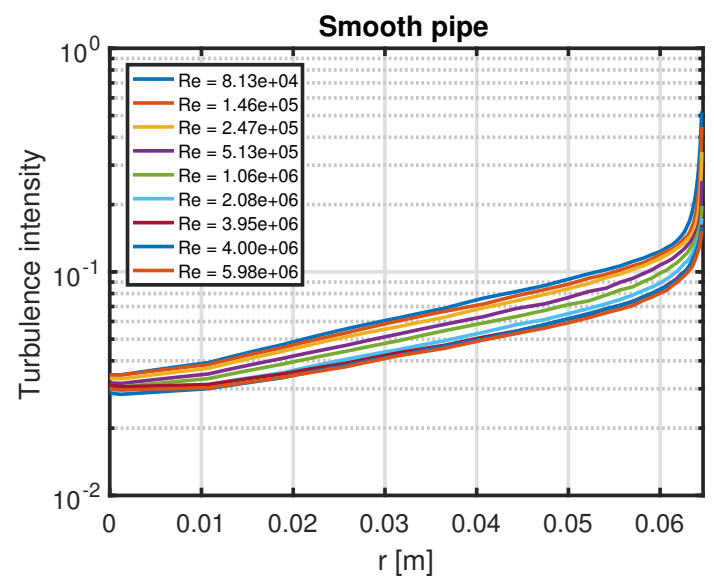

(a)

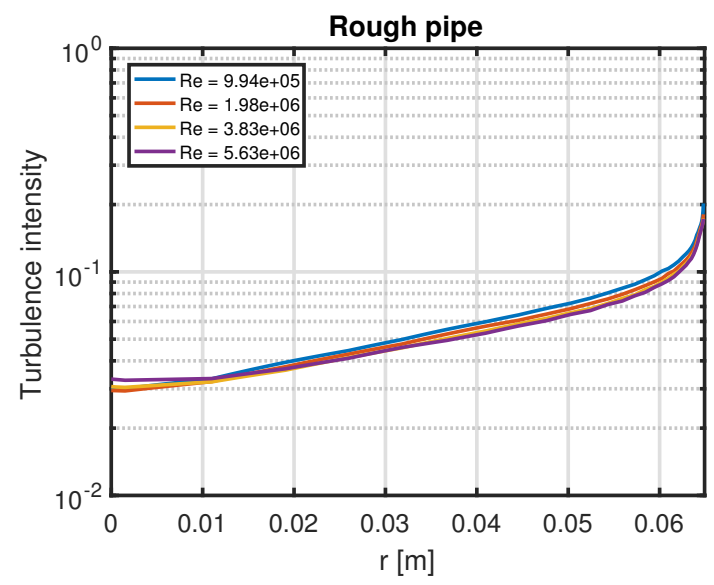

(b)

Figure 1. Turbulence intensity as a function of pipe radius, (a): smooth pipe; (b): rough pipe.

To make a direct comparison of the smooth and rough pipe measurements, we interpolate the smooth pipe measurements to the four $R e$ values where the rough pipe measurements are done. Furthermore, we use a normalized pipe radius $r_{n}=r / R$ to account for the difference in smooth and rough pipe radii. The result is a comparison of the TI profiles at four $R e$ (see Figure 2). As Re increases, we observe that the rough pipe TI becomes larger than the smooth pipe TI.

To make the comparison more quantitative, we define the turbulence intensity ratio (TIR):

$$
r_{I, \text { Rough } / \text { Smooth }}\left(r_{n}\right)=\frac{I_{\text {Rough }}\left(r_{n}\right)}{I_{\text {Smooth }}\left(r_{n}\right)}=\frac{v_{\text {RMS }, \text { Rough }}\left(r_{n}\right)}{v_{\text {RMS,Smooth }}\left(r_{n}\right)} \times \frac{v_{\text {Smooth }}\left(r_{n}\right)}{v_{\text {Rough }}\left(r_{n}\right)} .
$$

The TIR is shown in Figure 3. The left-hand plot shows all radii; prominent features are:

- $\quad$ The TIR on the axis is roughly one except for the highest $R e$, where it exceeds 1.1.

- In the intermediate region between the axis and the wall, an increase is already visible for the second-lowest $\operatorname{Re}, 1.98 \times 10^{6}$.

The events close to the wall are most clearly seen in the right-hand plot of Figure 3. A local peak of TIR is observed for all $R e$; the magnitude of the peak increases with $R e$. Note that we only analyse data to $99.8 \%$ of the pipe radius. Thus, the $0.13 \mathrm{~mm}$ closest to the wall is not considered.

The TIR information can also be represented by studying the TIR at fixed $r_{n}$ vs. $R e$ (see Figure 4). From this plot, we find that the magnitude of the peak close to the wall $\left(r_{n}=0.99\right)$ increases linearly with Re:

$$
r_{I, \text { Rough } / \text { Smooth }}\left(r_{n}=0.99\right)=2.5137 \times 10^{-8} \times R e+1.0161 \text {. }
$$




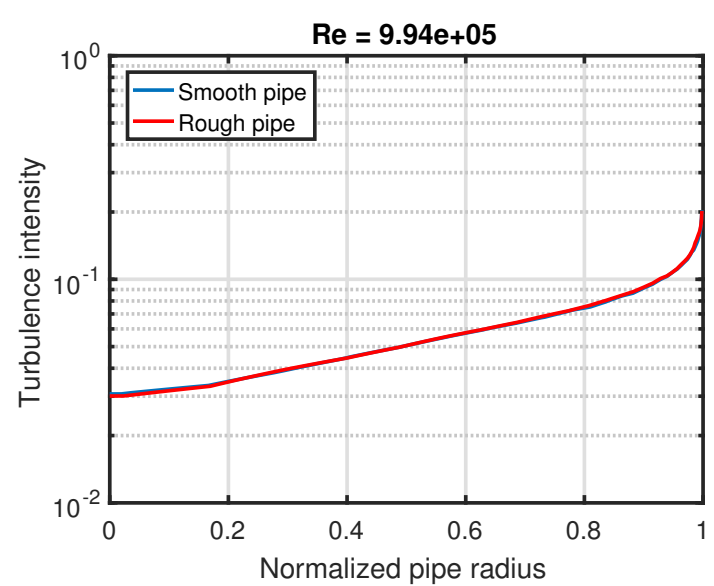

(a)

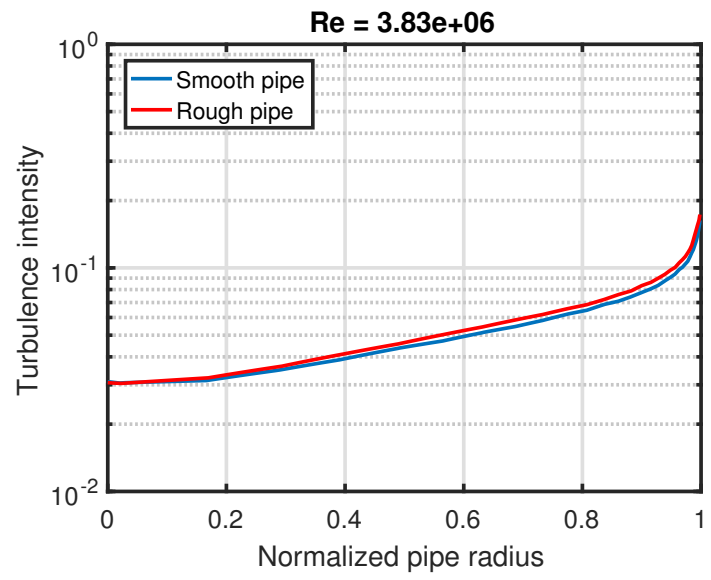

(c)

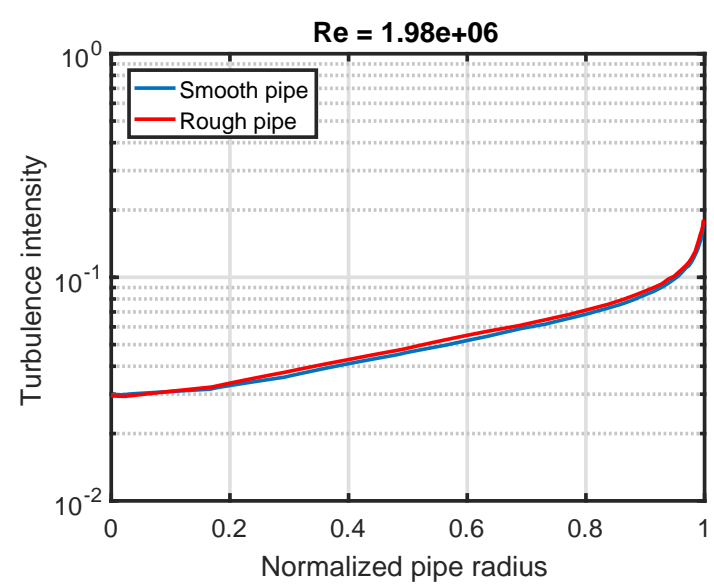

(b)

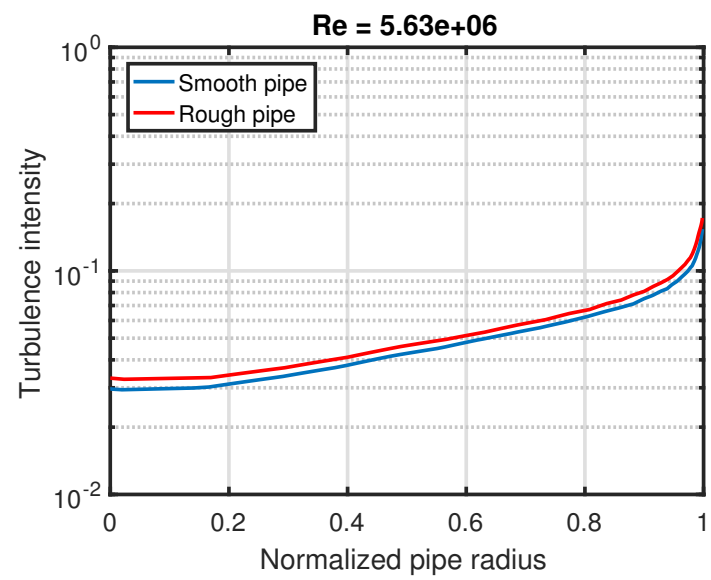

(d)

Figure 2. Comparison of smooth and rough pipe turbulence intensity (TI) profiles for the four $R e$ values where the rough pipe measurements are done, $(\mathbf{a}): \operatorname{Re}=9.94 \mathrm{e}+05 ;(\mathbf{b}): \operatorname{Re}=1.98 \mathrm{e}+06$; (c): $\operatorname{Re}=3.83 e+06 ;(d): \operatorname{Re}=5.63 e+06$.

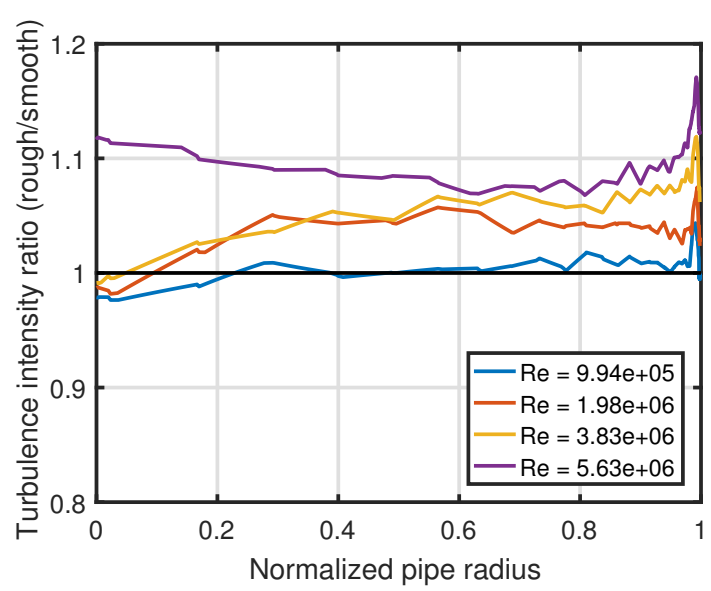

(a)

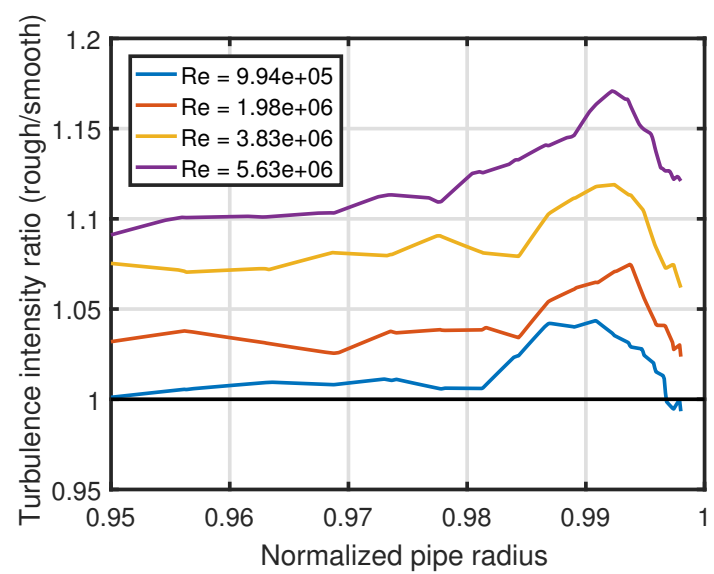

(b)

Figure 3. Turbulence intensity ratio (TIR); (a): all radii; (b): zoom to outer 5\%.

Information on fits of the TI profiles to analytical expressions can be found in Appendix A. 
Based on uncertainties in Table 2 in [3], the uncertainty of TI for the smooth (rough) pipe is $2.9 \%$ $(3.5 \%)$, respectively. Note that we have used $4.4 \%$ instead of $4.7 \%$ for the uncertainty of $v_{\mathrm{RMS}}^{2} / v_{\tau}^{2}$ to derive the rough pipe uncertainty. The resulting TIR uncertainty is $4.5 \%$.

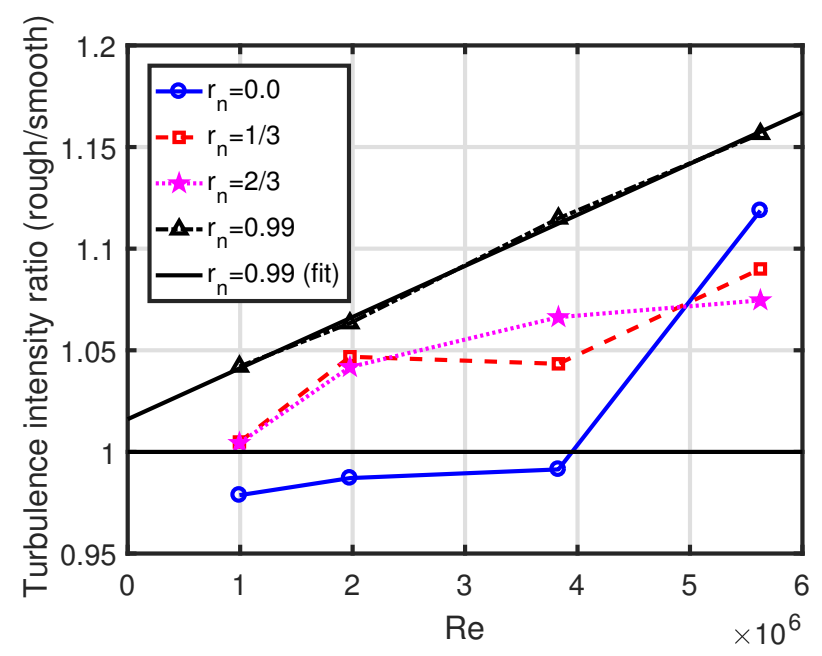

Figure 4. Turbulence intensity ratios for fixed $r_{n}$.

\section{Turbulence Intensity Scaling}

We define the TI averaged over the pipe area as:

$$
I_{\text {Pipe area }}=\frac{2}{R^{2}} \int_{0}^{R} \frac{v_{\mathrm{RMS}}(r)}{v(r)} r \mathrm{~d} r .
$$

In [5], another definition was used for the TI averaged over the pipe area. Analysis presented in Sections 3 and 4 is repeated using that definition in Appendix B.

Scaling of the TI with Re for smooth- and rough-wall pipe flow is shown in Figure 5. For $\operatorname{Re}=10^{6}$, the smooth and rough pipe values are almost the same. However, when Re increases, the TI of the rough pipe increases compared to the smooth pipe; this increase is to a large extent caused by the TI increase in the intermediate region between the pipe axis and the pipe wall (see Figures 3 and 4). We have not made fits to the rough wall pipe measurements because of the limited number of datapoints.

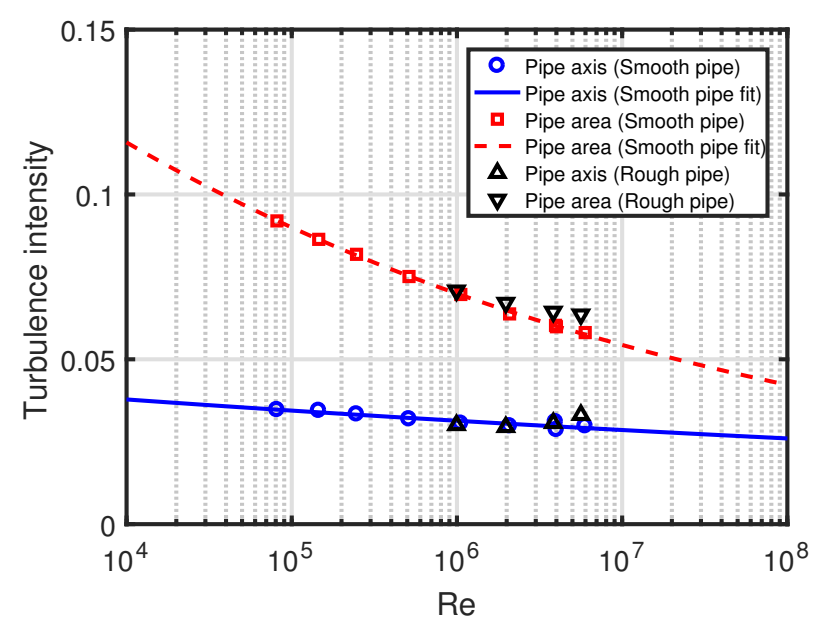

Figure 5. Turbulence intensity for smooth and rough pipe flow. 


\section{Friction Factor}

The fits shown in Figure 5 are:

$$
\begin{aligned}
& I_{\text {Smooth pipe axis }}=0.0550 \times R e^{-0.0407}, \\
& I_{\text {Smooth pipe area }}=0.317 \times R e^{-0.110} .
\end{aligned}
$$

The Blasius smooth pipe (Darcy) friction factor [12] is also expressed as an Re power-law:

$$
\lambda_{\text {Blasius }}=0.3164 \times R e^{-0.25} .
$$

The Blasius friction factor matches measurements best for $R e<10^{5}$; the friction factor by e.g., Gersten (Equation (1.77) in [13]) is preferable for larger Re. The Blasius and Gersten friction factors are compared in Figure 6 . The deviation between the smooth and rough pipe Gersten friction factors above $R e=10^{5}$ is qualitatively similar to the deviation between the smooth and rough pipe area TI in Figure 5. For the Gersten friction factors, we have used the measured pipe roughnesses.

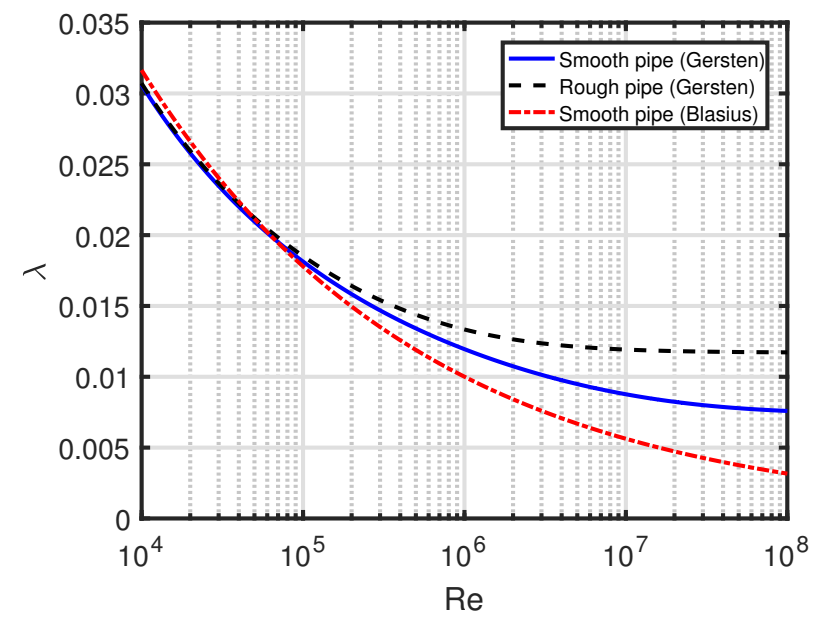

Figure 6. Friction factor.

For the smooth pipe, we can combine Equations (5) and (6) to relate the pipe area TI to the Blasius friction factor:

$$
\begin{array}{ll}
I_{\text {Smooth pipe area }} & =0.526 \times \lambda_{\text {Blasius' }}^{0.44} \\
\lambda_{\text {Blasius }} & =4.307 \times I_{\text {Smooth pipe area }}^{2.27} .
\end{array}
$$

The TI and Blasius friction factor scaling is shown in Figure 7.

For axisymmetric flow in the streamwise direction, the mean flow velocity averaged over the pipe area is:

$$
v_{m}=\frac{2}{R^{2}} \times \int_{0}^{R} v(r) r \mathrm{~d} r
$$

Now, we are in a position to define an average velocity of the turbulent fluctuations:

$$
\left\langle v_{\mathrm{RMS}}\right\rangle=v_{m} I_{\text {Pipe area }}=\frac{4}{R^{4}} \int_{0}^{R} v(r) r \mathrm{~d} r \int_{0}^{R} \frac{v_{\mathrm{RMS}}(r)}{v(r)} r \mathrm{~d} r .
$$

The friction velocity is:

$$
v_{\tau}=\sqrt{\tau_{w} / \rho},
$$

where $\tau_{w}$ is the wall shear stress and $\rho$ is the fluid density. 
The relationship between $\left\langle v_{\mathrm{RMS}}\right\rangle$ and $v_{\tau}$ is illustrated in Figure 8. From the fit, we have:

$$
\left\langle v_{\mathrm{RMS}}\right\rangle=1.8079 \times v_{\tau},
$$

which we approximate as:

$$
\left\langle v_{\text {RMS }}\right\rangle \sim \frac{9}{5} \times v_{\tau} .
$$

Equations (11) and (12) above correspond to the usage of the friction velocity as a proxy for the velocity of the turbulent fluctuations [14]. We note that the rough wall velocities are higher than for the smooth wall.

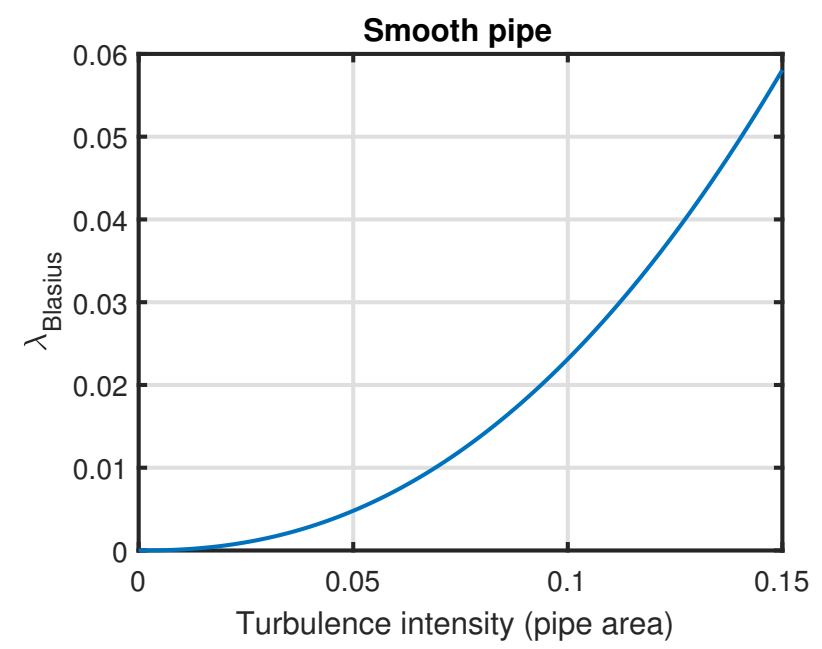

Figure 7. Relationship between pipe area turbulence intensity and the Blasius friction factor.

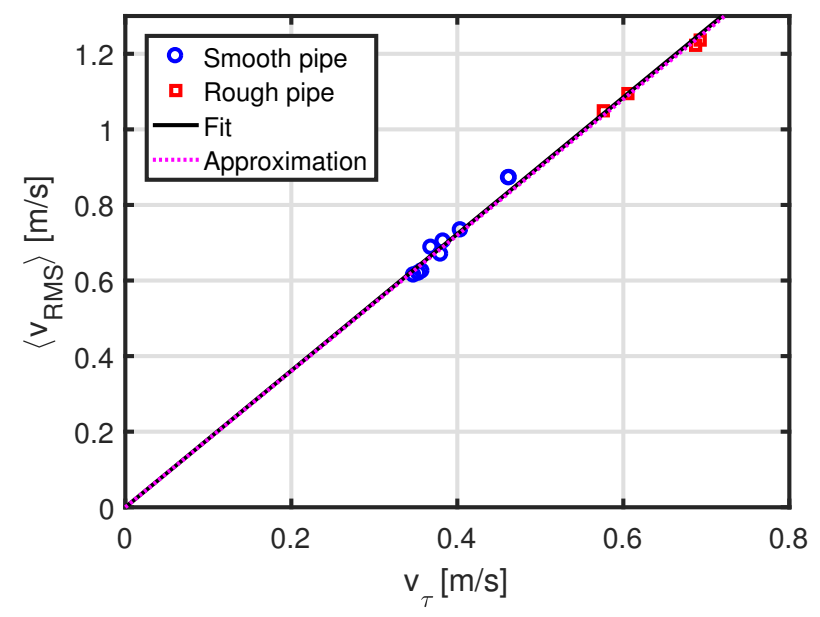

Figure 8. Relationship between friction velocity and the average velocity of the turbulent fluctuations.

Equations (9) and (12) can be combined with Equation (1.1) in [15]:

$$
\lambda=\frac{4 \tau_{w}}{\frac{1}{2} \rho v_{m}^{2}}=\frac{-(\Delta P / L) D}{\frac{1}{2} \rho v_{m}^{2}}=8 \times \frac{v_{\tau}^{2}}{v_{m}^{2}} \sim \frac{200}{81} \times I_{\text {Pipe area }}^{2}
$$

where $\Delta P$ is the pressure loss and $L$ is the pipe length. This can be reformulated as:

$$
I_{\text {Pipe area }} \sim \frac{9}{10 \sqrt{2}} \times \sqrt{\lambda} .
$$


We show how well this approximation works in Figure 9. Overall, the agreement is within $15 \%$.

We proceed to define the average kinetic energy of the turbulent velocity fluctuations $\left\langle E_{\text {kin,RMS }}\right\rangle$ (per pipe volume $V$ ) as:

$$
\begin{aligned}
\left\langle E_{\mathrm{kin}, \mathrm{RMS}}\right\rangle / V & =\frac{1}{2} \rho\left\langle v_{\mathrm{RMS}}\right\rangle^{2} \sim-\frac{81}{50} \times(\Delta P / L) D / 4 \\
& =\frac{81}{50} \times \tau_{w}=\frac{81}{50} \times v_{\tau}^{2} \rho,
\end{aligned}
$$

with $V=L \pi R^{2}$, so we have:

$$
\begin{aligned}
\left\langle E_{\mathrm{kin}, \mathrm{RMS}}\right\rangle & =\frac{1}{2} m\left\langle v_{\mathrm{RMS}}\right\rangle^{2} \sim-\frac{81}{50} \times(\pi / 2) R^{3} \Delta P \\
& =\frac{81}{50} \times \tau_{w} V=\frac{81}{50} \times v_{\tau}^{2} m,
\end{aligned}
$$

where $m$ is the fluid mass. The pressure loss corresponds to an increase of the turbulent kinetic energy. The turbulent kinetic energy can also be expressed in terms of the mean flow velocity and the TI or the friction factor:

$$
\left\langle v_{\mathrm{RMS}}\right\rangle^{2}=v_{m}^{2} I_{\text {Pipe area }}^{2} \sim \frac{81}{200} \times v_{m}^{2} \lambda
$$

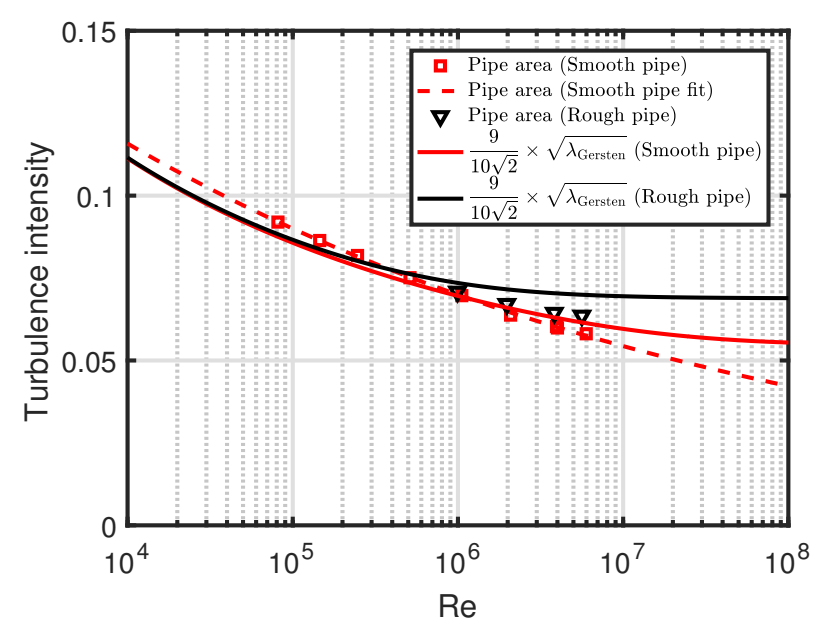

Figure 9. Turbulence intensity for smooth and rough pipe flow. The approximation in Equation (14) is included for comparison.

\section{Discussion}

\subsection{The Attached Eddy Hypothesis}

Our quantification of the ratio $\left\langle v_{\mathrm{RMS}}\right\rangle / v_{\tau}$ as a constant can be placed in the context of the attached eddy hypothesis by Townsend [16,17]. Our results are for quantities averaged over the pipe radius, whereas the attached eddy hypothesis provides a local scaling with distance from the wall. By proposing an overlap region (see Figure 1 in [18]) between the inner and outer scaling [19], it can be deduced that $\left\langle v_{\mathrm{RMS}}\right\rangle / v_{\tau}$ is a constant in this overlap region [20,21]. Such an overlap region has been shown to exist in $[2,20]$. The attached eddy hypothesis has provided the basis for theoretical work on e.g., the streamwise turbulent velocity fluctuations in flat-plate [22] and pipe flow [23] boundary layers. Work on the law of the wake in wall turbulence also makes use of the attached eddy hypothesis [24].

As a consistency check for our results, we can compare the constant 9/5 in Equation (12) to the prediction by Townsend:

$$
\frac{v_{\mathrm{RMS}, \text { Townsend }}(r)^{2}}{v_{\tau}^{2}}=B_{1}-A_{1} \ln \left(\frac{R-r}{r}\right),
$$

where fits have provided the constants $B_{1}=1.5$ and $A_{1}=1.25$. Here, $A_{1}$ is a universal constant, whereas $B_{1}$ is not expected to be a constant for different wall-bounded flows [25]. The constants are 
averages of fits presented in [3] to the smooth- and rough-wall Princeton Superpipe measurements. The Townsend-Perry constant $A_{1}$ was found to be 1.26 in [25]. Performing the area averaging yields:

$$
\frac{\left\langle v_{\mathrm{RMS}, \text { Townsend }}\right\rangle^{2}}{v_{\tau}^{2}}=B_{1}+\frac{3}{2} \times A_{1}=3.38
$$

Our finding is:

$$
\frac{\left\langle v_{\mathrm{RMS}}\right\rangle^{2}}{v_{\tau}^{2}} \sim\left(\frac{9}{5}\right)^{2}=3.24
$$

which is within 5\% of the result in Equation (19). The reason that our result is smaller is that Equation (18) is overpredicting the turbulence level close to the wall and close to the pipe axis. Equation (18) as an upper bound has also been discussed in [26].

\subsection{The Friction Factor and Turbulent Velocity Fluctuations}

The proportionality between the average kinetic energy of the turbulent velocity fluctuations and the friction velocity squared has been identified in [27] for $R e>10^{5}$. This corresponds to our Equation (16).

A correspondence between the wall-normal Reynolds stress and the friction factor has been shown in [28]. Those results were found using DNS. The main difference between the cases is that we use the streamwise Reynolds stress. However, for an eddy rotating in the streamwise direction, both a wall-normal and a streamwise component should exist which connects the two observations.

\subsection{The Turbulence Intensity and the Diagnostic Plot}

Other related work can be found beginning with [29] where the diagnostic plot was introduced. In the following publications, a version of the diagnostic plot was brought forward where the local TI is plotted as a function of the local streamwise velocity normalised by the free stream velocity [30-32]. Equation (3) in [31] corresponds to our $I_{\text {Core }}$ (see Equation (A1) in Appendix A).

\subsection{Applicability of Turbulence Intensity Scaling with Friction Factor}

The scaling of TI with the friction factor (Equation (14)) was found based on pipe flow measurements with two roughnesses. It is an open question whether our result holds in the fully rough regime. For the fully rough regime, the friction factor becomes a constant for high $R e$. As a consequence of our scaling expression, this should also be the case for the TI.

It is clear that the specific formula is not directly applicable for other wall-bounded flows, since $B_{1}$ takes different values. However, the basic behaviour, i.e., that the TI scales with the square root of the friction factor, may be universally valid.

\section{Conclusions}

We have compared TI profiles for smooth- and rough-wall pipe flow measurements made in the Princeton Superpipe.

The change of the TI profile with increasing Re from hydraulically smooth to fully rough flow exhibits propagation from the pipe wall to the pipe axis. The TIR at $r_{n}=0.99$ scales linearly with $R e$.

The scaling of TI with $R e$ - on the pipe axis and averaged over the pipe area-shows that the smooth- and rough-wall level deviates with increasing Reynolds number.

We find that $I_{\text {Pipe area }} \sim \frac{9}{10 \sqrt{2}} \times \sqrt{\lambda}$. This relationship can be useful to calculate the TI given a known $\lambda$, both for smooth and rough pipes. It follows that given a pressure loss in a pipe, the turbulent kinetic energy increase can be estimated.

Acknowledgments: We thank Alexander J. Smits for making the Superpipe data publicly available.

Conflicts of Interest: The authors declare no conflict of interest. 


\section{Appendix A. Fits to the Turbulence Intensity Profile}

As we have done for the smooth pipe measurements in [5], we can also fit the rough pipe measurements to this function:

$$
\begin{aligned}
I\left(r_{n}\right) & =I_{\text {Core }}\left(r_{n}\right)+I_{\text {Wall }}\left(r_{n}\right) \\
& =\left[\alpha+\beta \times r_{n}^{\gamma}\right]+\left[\delta \times\left|\ln \left(1-r_{n}\right)\right|^{\varepsilon}\right],
\end{aligned}
$$

where $\alpha, \beta, \gamma, \delta$ and $\varepsilon$ are fit parameters. A comparison of fit parameters found for the smooth- and rough-pipe measurements is shown in Figure A1. Overall, we can state that the fit parameters for the smooth and rough pipes are in a similar range for $10^{6}<\operatorname{Re}<6 \times 10^{6}$.

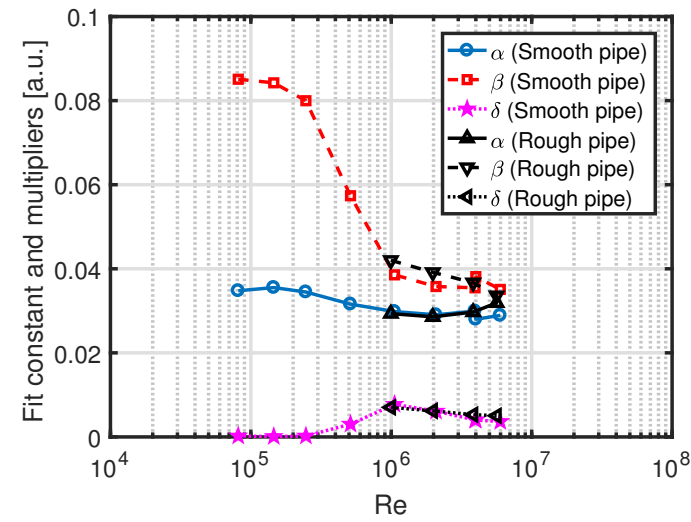

(a)

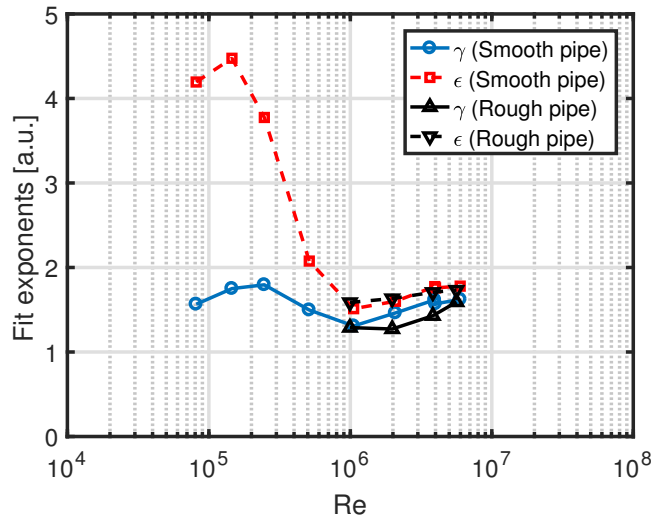

(b)

Figure A1. Comparison of smooth- and rough-pipe fit parameters, (a): fit parameters $\alpha, \beta$ and $\delta$; (b): fit parameters $\gamma$ and $\varepsilon$.

The $\min / \max$ deviation of the rough pipe fit from the measurements is below $10 \%$; see the comparison to the smooth wall fit min/max deviation in Figure A2.

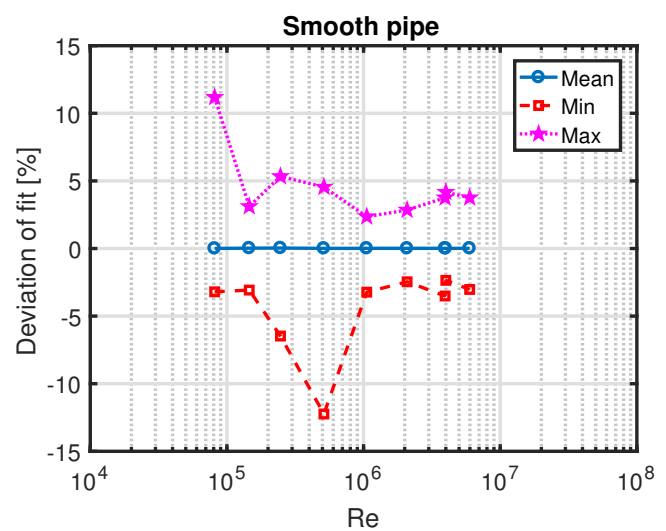

(a)

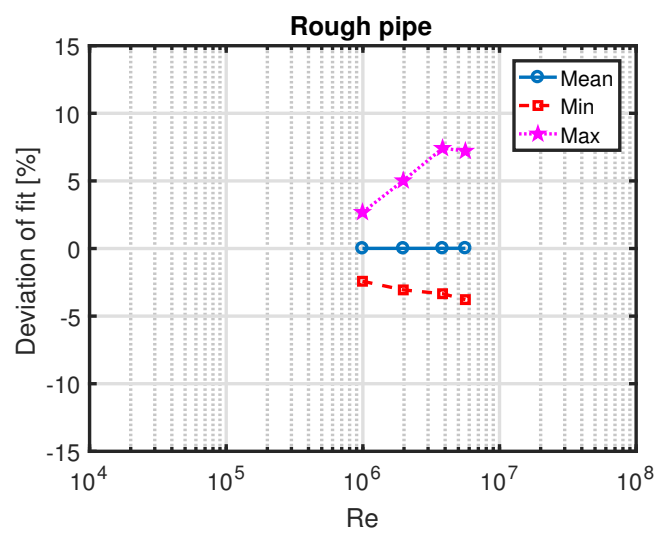

(b)

Figure A2. Deviation of fits to measurements; (a): smooth pipe, (b): rough pipe.

The core and wall fits for the smooth and rough pipe fits are compared in Figure A3. 


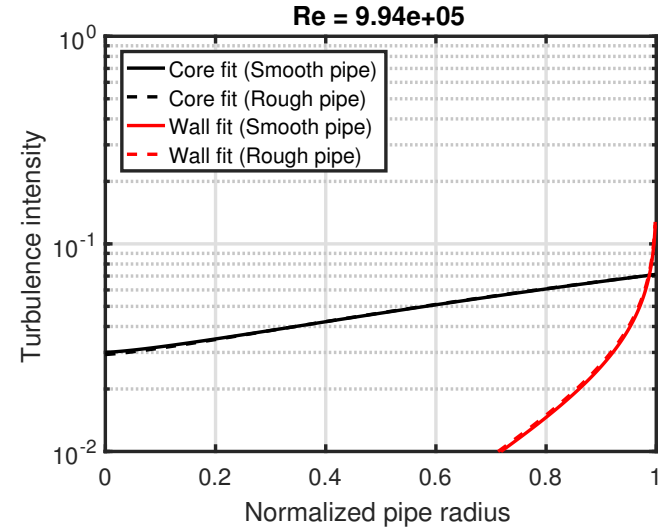

(a)

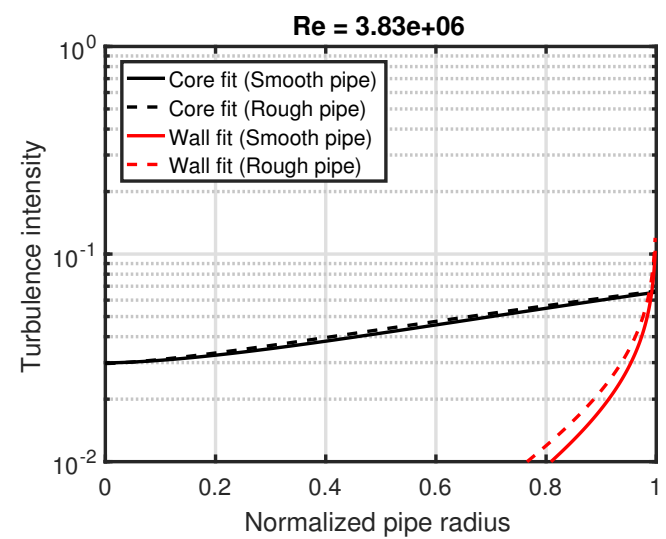

(c)

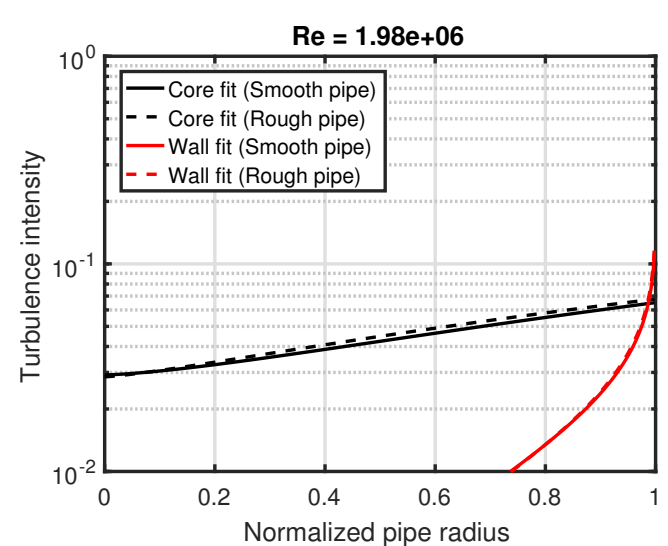

(b)

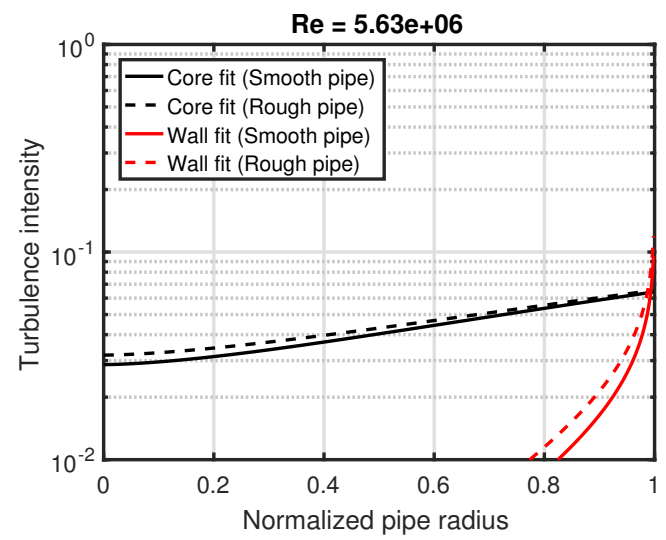

(d)

Figure A3. Comparison of smooth and rough pipe core and wall fits, (a): $\operatorname{Re}=9.94 \mathrm{e}+05$; (b): $\operatorname{Re}=1.98 \mathrm{e}+06 ;(\mathbf{c}): \operatorname{Re}=3.83 \mathrm{e}+06 ;(\mathbf{d}): \operatorname{Re}=5.63 \mathrm{e}+06$.

The position where the core and wall TI levels are equal is shown in Figure A4. This position does not change significantly for the rough pipe; however, the position does increase with $R e$ for the smooth pipe: this indicates that the wall term becomes less important relative to the core term.

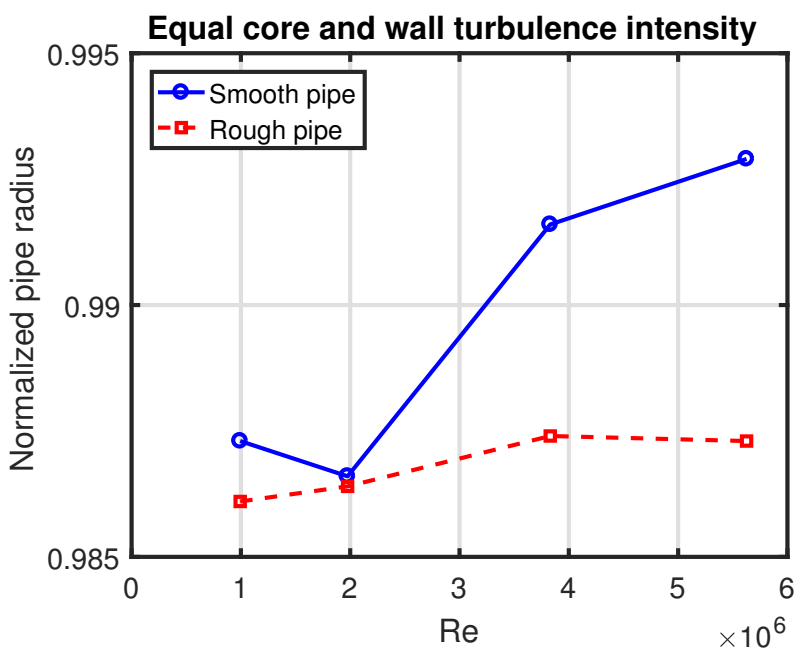

Figure A4. Normalised pipe radius where the core and wall TI levels are equal. 


\section{Appendix B. Arithmetic Mean Definition of Turbulence Intensity Averaged Over the Pipe Area}

In the main paper, we have defined the TI over the pipe area in Equation (4). In [5], we used the arithmetic mean (AM) instead:

$$
I_{\text {Pipe area, } \mathrm{AM}}=\frac{1}{R} \int_{0}^{R} \frac{v_{\mathrm{RMS}}(r)}{v(r)} \mathrm{d} r .
$$

The AM leads to a somewhat different pipe area scaling for the smooth pipe measurements, which is illustrated in Figure A5. Compare to Figure 5.

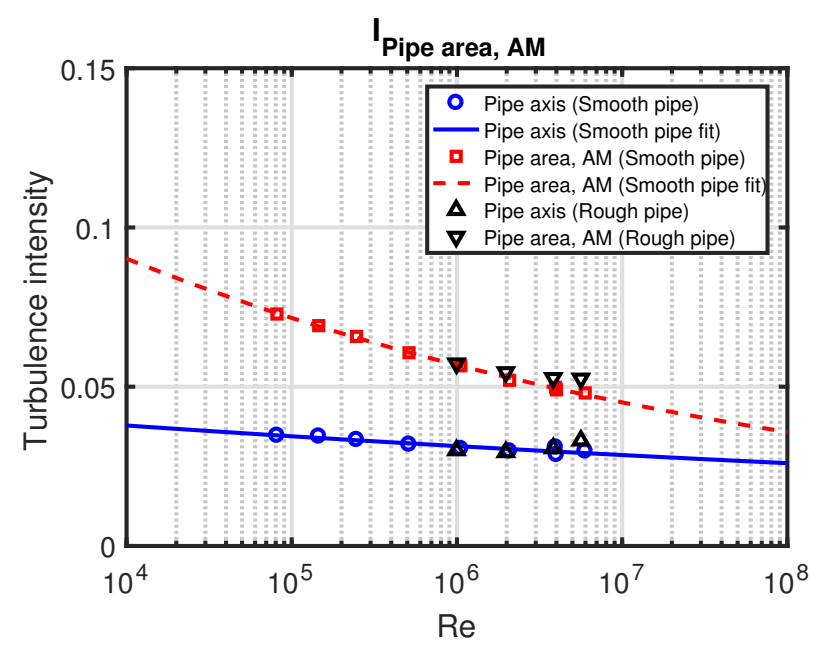

Figure A5. Turbulence intensity for smooth and rough pipe flow. The arithmetic mean (AM) is used for the pipe area TI.

The scaling found in [5] using this definition is:

$$
I_{\text {Smooth pipe area, } \mathrm{AM}}=0.227 \times R e^{-0.100} .
$$

The AM scaling also has implications for the relationship with the Blasius friction factor scaling (Equation (7)):

$$
\begin{array}{ll}
I_{\text {Smooth pipe area, } \mathrm{AM}} & =0.360 \times \lambda_{\text {Blasius' }}^{0.4} \\
\lambda_{\text {Blasius }} & =12.89 \times I_{\text {Smooth pipe area, }}^{2.5} \text { AM }
\end{array}
$$

We can now define the AM version of the average velocity of the turbulent fluctuations:

$$
\left\langle v_{\mathrm{RMS}}\right\rangle_{\mathrm{AM}}=v_{m} I_{\text {Pipe area, } \mathrm{AM}}=\frac{2}{R^{3}} \int_{0}^{R} v(r) r \mathrm{~d} r \int_{0}^{R} \frac{v_{\mathrm{RMS}}(r)}{v(r)} \mathrm{d} r .
$$

The AM definition can be considered as a first order moment equation for $v_{\mathrm{RMS}}$, whereas the definition in Equation (9) is a second order moment equation.

Again, we find that the AM average turbulent velocity fluctuations are proportional to the friction velocity. However, the constant of proportionality is different than the one in Equation (11) (see Figure A6). The AM case can be fitted as:

$$
\left\langle v_{\mathrm{RMS}}\right\rangle_{\mathrm{AM}}=1.4708 \times v_{\tau},
$$

which we approximate as: 


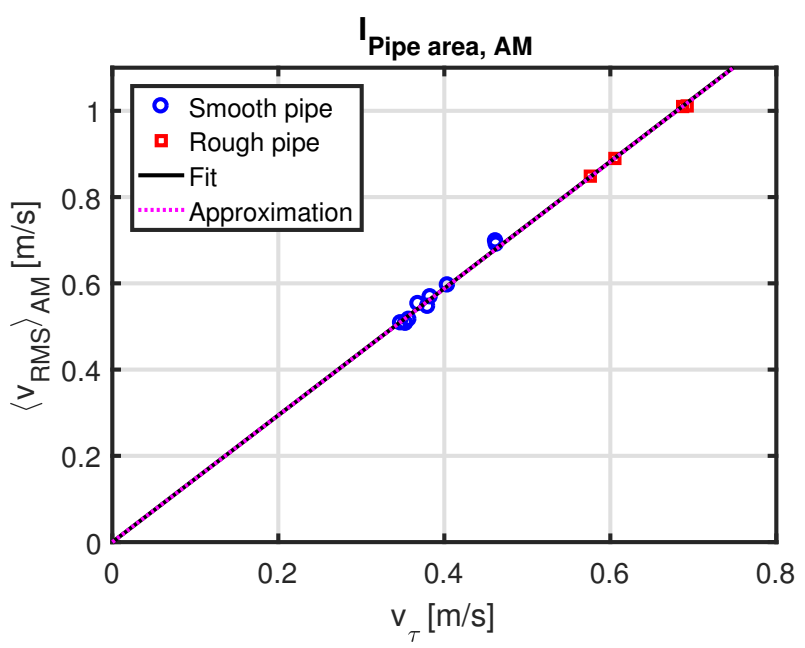

Figure A6. Relationship between friction velocity and the AM average velocity of the turbulent fluctuations.

As we did in Section 5, we can perform the AM averaging of Equation (18) (also done in [26]):

$$
\frac{\left\langle v_{\mathrm{RMS}, \text { Townsend }}\right\rangle_{\mathrm{AM}}^{2}}{v_{\tau}^{2}}=B_{1}+A_{1}=2.75,
$$

where we find:

$$
\frac{\left\langle v_{\mathrm{RMS}}\right\rangle_{\mathrm{AM}}^{2}}{v_{\tau}^{2}} \sim \frac{2}{3} \times\left(\frac{9}{5}\right)^{2}=2.16 .
$$

\section{References}

1. Marusic, I.; McKeon, B.J.; Monkewitz, P.A.; Nagib, H.M.; Smits, A.J.; Sreenivasan, K.R. Wall-bounded turbulent flows at high Reynolds numbers: Recent advances and key issues. Phys. Fluids 2010, 22, 065103.

2. Hultmark, M.; Vallikivi, M.; Bailey, S.C.C.; Smits, A.J. Turbulent pipe flow at extreme Reynolds numbers. Phys. Rev. Lett. 2012, 108, 094501.

3. Hultmark, M.; Vallikivi, M.; Bailey, S.C.C.; Smits, A.J. Logarithmic scaling of turbulence in smooth- and rough-wall pipe flow. J. Fluid Mech. 2013, 728, 376-395.

4. Princeton Superpipe. 2017. Available online: https://smits.princeton.edu/superpipe-turbulence-data/ (accessed on 7 June 2017).

5. Russo, F.; Basse, N.T. Scaling of turbulence intensity for low-speed flow in smooth pipes. Flow Meas. Instrum. 2016, 52, 101-114.

6. Langelandsvik, L.I.; Kunkel, G.J.; Smits, A.J. Flow in a commercial steel pipe. J. Fluid Mech. 2008, 595, 323-339.

7. Nikuradse, J. Strömungsgesetze in Rauhen Rohren; Springer-VDI-Verlag GmbH: Düsseldorf, Germany, 1933.

8. Jiménez, J. Turbulent flows over rough walls. Annu. Rev. Fluid Mech. 2004, 36, 173-196.

9. Flack, K.A.; Schultz, M.P. Review of hydraulic roughness scales in the fully rough regime. J. Fluids Eng. 2010, 132, 041203.

10. Chan, L.; MacDonald, M.; Chung, D.; Hutchins, N.; Ooi, A. A systematic investigation of roughness height and wavelength in turbulent pipe flow in the transitionally rough regime. J. Fluid Mech. 2015, 771, 743-777.

11. ANSYS Fluent User's Guide, Release 18.0; 2017. Available online: http://www.ansys.com/products/fluids / (accessed on 7 June 2017).

12. Blasius, H. Das Ähnlichkeitsgesetz bei Reibungsvorgängen in Flüssigkeiten; Springer-VDI-Verlag GmbH: Düsseldorf, Germany, 1913; pp. 1-40. 
13. Gersten, K. Fully developed turbulent pipe flow. In Fluid Mechanics of Flow Metering; Merzkirch, W., Ed.; Springer: Berlin, Germany, 2005.

14. Schlichting, H.; Gersten, K. Boundary-Layer Theory, 8th ed.; Springer: Berlin, Germany, 2000.

15. McKeon, B.J.; Zagarola, M.V.; Smits, A.J. A new friction factor relationship for fully developed pipe flow. J. Fluid Mech. 2005, 538, 429-443.

16. Townsend, A.A. The Structure of Turbulent Shear Flow, 2nd ed.; Cambridge University Press: Cambridge, UK, 1976.

17. Marusic, I.; Nickels, T.N.A.A. Townsend. In A Voyage through Turbulence; Davidson, P.A., Kaneda, Y., Moffatt, K., Sreenivasan, K.R., Eds.; Cambridge University Press: Cambridge, UK, 2011.

18. McKeon, B.J.; Morrison, J.F. Asymptotic scaling in turbulent pipe flow. Phil. Trans. Royal Soc. A 2007, 365, 771-787.

19. Millikan, C.B. A critical discussion of turbulent flows in channels and circular tubes. In Proceedings of the 5th International Congress for Applied Mechanics, New York, NY, USA, 12-16 September 1938.

20. Perry, A.E.; Abell, C.J. Scaling laws for pipe-flow turbulence. J. Fluid Mech. 1975, 67, 257-271.

21. Perry, A.E.; Abell, C.J. Asymptotic similarity of turbulence structures in smooth- and rough-walled pipes. J. Fluid Mech. 1977, 79, 785-799.

22. Marusic, I.; Kunkel, G.J. Streamwise turbulence intensity formulation for flat-plate boundary layers. Phys. Fluids 2003, 15, 2461-2464.

23. Hultmark, M. A theory for the streamwise turbulent fluctuations in high Reynolds number pipe flow. J. Fluid Mech. 2012, 707, 575-584.

24. Krug, D.; Philip, K.; Marusic, I. Revisiting the law of the wake in wall turbulence. J. Fluid Mech. 2017, 811, 421-435.

25. Marusic, I.; Monty, J.P.; Hultmark, M.; Smits, A.J. On the logarithmic region in wall turbulence. JFM Rapids 2013, 716, R3.

26. Pullin, D.I.; Inoue, M.; Saito, N. On the asymptotic state of high Reynolds number, smooth-wall turbulent flows. Phys. Fluids 2013, 25, 015116.

27. Yakhot, V.; Bailey, S.C.C.; Smits, A.J. Scaling of global properties of turbulence and skin friction in pipe and channel flows. J. Fluid Mech. 2010, 652, 65-73.

28. Orlandi, P. The importance of wall-normal Reynolds stress in turbulent rough channel flows. Phys. Fluids 2013, 25, 110813.

29. Alfredsson, P.H.; Örlü, R. The diagnostic plot-A litmus test for wall bounded turbulence data. Eur. J. Mech. $B$ Fluids 2010, 29, 403-406.

30. Alfredsson, P.H.; Segalini, A.; Örlü, R. A new scaling for the streamwise turbulence intensity in wall-bounded turbulent flows and what it tells us about the "outer" peak. Phys. Fluids 2011, 23, 041702.

31. Alfredsson, P.H.; Örlü, R.; Segalini, A. A new formulation for the streamwise turbulence intensity distribution in wall-bounded turbulent flows. Eur. J. Mech. B Fluids 2012, 36, 167-175.

32. Castro, I.P.; Segalini, A.; Alfredsson, P.H. Outer-layer turbulence intensities in smooth- and rough-wall boundary layers. J. Fluid Mech. 2013, 727, 119-131.

(C) 2017 by the authors. Licensee MDPI, Basel, Switzerland. This article is an open access article distributed under the terms and conditions of the Creative Commons Attribution (CC BY) license (http://creativecommons.org/licenses/by/4.0/). 